\title{
Temporal trends in loss of life expectancy after a cancer diagnosis among the Australian
}

\section{population}

Kou Kou ${ }^{1,2}$, Paramita Dasgupta ${ }^{1}$, Susanna Cramb ${ }^{1,2}$, Xue Qin $\mathrm{Yu}^{3,4}$, Therese Andersson ${ }^{5}$, Peter Baade ${ }^{1,6,7, \S}$

${ }^{1}$ Cancer Research Centre, Cancer Council Queensland, Brisbane, Australia

${ }^{2}$ Institute of Health and Biomedical Innovation, School of Public Health and Social Work, Queensland University of Technology, Kelvin Grove, Brisbane QLD 4059, Australia

${ }^{3}$ Cancer Research Division, Cancer Council New South Wales, Kings Cross, Sydney NSW 1340 Australia

${ }^{4}$ Sydney School of Public Health, Faculty of Medicine and Health, University of Sydney, NSW 2006, Australia.

${ }^{5}$ Department of Medical Epidemiology and Biostatistics, Karolinska Institutet, Stockholm, Sweden.

${ }^{6}$ Menzies Health Institute Queensland, Griffith University, Gold Coast Campus, Parklands Drive, Southport QLD 4222, Australia

${ }^{7}$ School of Mathematical Sciences, Queensland University of Technology, Gardens Point, Brisbane QLD 4000, Australia

\section{$\S$ Corresponding Author}

Prof Peter D Baade, Senior Research Fellow

Cancer Council Queensland, PO Box 201, Spring Hill QLD 4001 Australia

Email: peterbaade@cancerqld.org.au

Fax: +61 73259 8527; Phone: +61 736345317

\section{Word count}

Abstract: 245; Manuscript: 3174; Highlights: 82 


\begin{abstract}
Background: Loss of life expectancy (LOLE) provides valuable insights into the impact of cancer. We evaluated the temporal trends in LOLE for Australian cancer patients and the gain in life years for recently diagnosed patients due to survival improvements.

Methods: Analysis was conducted using an Australian population-based cohort ( $\mathrm{n}=$ $1,865,154)$ aged 50-89 years, who were primarily diagnosed with one of 19 leading cancers between 1982-2015. Flexible parametric survival models were used to estimate LOLE and the proportion of life lost (POLL) by year, age group, sex, and, for New South Wales only, spread of disease. The total years of LOLE and gain in life years due to survival improvements were estimated for those diagnosed in 2014.

Results: For 19 cancers combined, LOLE and POLL were significantly lower for more recent diagnoses. Cancer-specific temporal trends were consistent by age, sex, and spread of disease (where relevant) although the magnitude varied. Prostate, kidney, or non-Hodgkin lymphoma experienced the largest decreases in POLL over time. For the 2014 diagnoses, an estimation of 403,094 life years lost will be caused by the 19 cancers. With the increase in cancer survival over time, the 2014 cohort will gain an extra 432,588 life years (52\%) compared to that experienced by the 1982 cohort.

Conclusion: While reduced impact of a cancer diagnosis on LOLE over time is encouraging, the growing number of cancer survivors in Australia is likely to pose complex challenges for cancer patients, their care givers, and health-care systems.
\end{abstract}

Key words: cancer; survival; life expectancy; temporal; Australia 


\section{Background}

Population survival statistics provide cancer patients with an insight into the implications of their diagnosis. While measures such as the relative survival rate are often used for this purpose [1], the survival rate at a certain time point after diagnosis (e.g. 10-year survival) fails to provide a complete picture of the survival outcomes. For example, while on average, people diagnosed with cancer at a younger age usually have better survival than older patients [2], younger people have more years of remaining life to lose.

A measure designed specifically to evaluate the impact of cancer on life expectancy, loss of life expectancy (LOLE) [3], reflects the difference between patients' life expectancy and the life expectancy of similar individuals from the general population [4]. A number of studies have reported contemporary LOLE estimates and changes over time [5-10], including for Australia $[4,11]$. The Australian studies reported that LOLE decreased with age at diagnosis, but increased with spread of disease at diagnosis, and had reduced between 1990 and 2000. However, these studies only considered six cancers and the time period covered is no longer contemporary. Here, we present the temporal trend in LOLE due to 19 leading cancers for patients diagnosed in Australia from 1982 to 2015.

\section{Methods}

Ethical approval was obtained from ACT Human Research Ethics Committee (ETHLR.16.228), the Queensland University of Technology Human Research Ethics Committee (1600000868), and NSW Population \& Health Services Research Ethics Committee (2016/HRE1203). De-identified data were obtained from the Australian Cancer Database (ACD) held by the Australian Institute of Health and Welfare (AIHW). In Australia, notification of invasive cancers (excluding keratinocyte cancers) to each of the population-based cancer registries that provide data to the ACD is required by law [12].

\subsection{Cancer cohort}

The cohort comprised Australians diagnosed with one of 19 leading cancers (see Table A.1 for ICD-10 codes) during 1982-2015 (up to 2014 for the state of New South Wales (NSW) only), with only the first primary cancer diagnosed included $(n=2,291,652)$. Vital status was determined through routine annual linkage of cancer records with the Australian National Death Index, with follow-up to 31 December 2015. Consistent with previous studies [9, 10, 13], patients diagnosed before 50 years of age or after 89 years $(n=391,416)$ were excluded. Cases diagnosed based on death certificate only $(n=24,286)$ or who survived less than one 
day $(\mathrm{n}=10,796)$ were also excluded, leaving 1,865,154 patients aged 50-89 years in the final cohort. Information on spread of disease at diagnosis, which is a broad categorisation of how far a cancer has spread from its point of origin [14], was only available for solid tumours diagnosed in NSW during 1982-2014 $(\mathrm{n}=568,117)$.

\subsection{Life expectancy}

Life expectancy for people diagnosed with cancer is calculated using the area under the observed (all-cause) survival curve [4]. Therefore, to estimate life expectancy, the follow-up interval for the cohort must be long enough that their observed survival reaches zero. This is often not achieved since follow-up is typically too short to observe all deaths in the cohort, particularly for younger people given their higher remaining life expectancy. For the remaining cohort, the observed survival curves were extrapolated beyond the observed data using the relationship between relative survival, observed survival, and population survival [15]. Relative survival curves were extrapolated using flexible parametric models [16]. Population survival was also estimated using the same model based on Australian population life tables stratified by year, age, and sex (or NSW population life table for the NSW cancer cohort). The cohort method was used to facilitate the assessment of temporal trends [13].

\subsection{Statistical measures}

Three statistical measures are reported: LOLE, quantifies the differences in life expectancy of the general population (population life expectancy) and that of cancer patients. Given the dependency of LOLE on age, a second related measure, the proportion of life lost (POLL), was calculated as LOLE divided by the population life expectancy [10]. The POLL measure has been shown to be more comparable across age [17]. LOLE and POLL were estimated by year of diagnosis. The third measure is the 'gain in life years', a concept recently developed to quantify the impact of survival differences between socioeconomic strata for female colon cancer in England [17]. In our study, 'gain in life years' quantifies the impact of relative survival improvements over time on the overall burden of life years lost for Australian cancer patients. Since the 2015 NSW data were not available, we estimated the 'gain in life years' for those diagnosed in 2014 in Australia, as the difference between the estimated life expectancy of the cohort diagnosed in 2014 and the life expectancy if this 2014 cohort had the same relative survival as those diagnosed in 1982.

\subsection{Statistical models}


Flexible parametric survival models capture the shape of the underlying hazard function using restricted cubic splines [18]. It is possible to make a variety of assumptions about the future excess mortality within the model framework $[13,16]$. Since the relative survival curves generated using the study cohort showed that statistical cure was reached within the observed study timeframe for all 19 cancers (unpublished data showed same pattern observed using an earlier cohort [19]), we used the statistical cure assumption for the survival curve extrapolations [16]. This assumption was applied by restricting the underlying hazard function to have zero slope after the last knot [20].

For each cancer, models included year, age, sex, and, for NSW data only, spread of disease. Year and age were included as continuous variables using restricted cubic splines with four and two degrees of freedom (df) respectively. Interactions between year and age were included. Likelihood ratio tests supported the inclusion of both year and age as time-varying effects. Depending on site, we used 3-8 df (2-7 internal 'knots') for the baseline and 2-5 df (1-4 internal 'knots') for time-varying effects, with the selection of the number of knots in each instance based on minimizing the Bayesian Information Criterion.

Cancer-specific models were used to predicted LOLE and POLL for each cancer type. Results for the overall age groups were standardised using the age distribution for the whole cohort. Estimates for the combined cancer types were generated by calculating a weighted average of the cancer-specific estimates, with the weights reflecting the distribution of these cancers over the whole study period.

Consistent with previous studies [9, 10], we included all cases up to the year of last follow up. The direction, magnitude and significance of linear temporal trends in the POLL were quantified using simple linear regression models, in which the outcome variable was the yearspecific POLL, and year of diagnosis was an explanatory variable.

The model predicted life expectancy after cancer diagnosis was used for calculating the 'gain in life years' (Figure 1). Specifically, for a given cancer diagnosed in 2014, the estimated life expectancy is the area under the observed survival curve in 2014 (survival curve $\alpha$ in Figure 1). In contrast, the area under the observed survival curve based on the 1982 relative survival and 2014 population survival estimates (survival curve $\beta$ ) represents the corresponding life expectancy if a person had the same cancer-specific survival as those diagnosed in 1982. Therefore, the area between survival curves $\alpha$ and $\beta$ (Shade B) is the 'gain in life years' due to the improvements in relative survival from 1982 to 2014 (Appendix C). 


\subsection{Sensitivity analysis}

With the introduction of different cancer screening programs and early detection methods in Australia during the $1990 \mathrm{~s}$, it is possible that the survival time for some screen detected cancers were inflated through lead-time bias. Lead-time is the length of time a diagnosis is advanced by screening [21]. For example, in a simulation study for breast cancer, with moderate screening sensitivity, the mean lead-time among screen detected breast cancer was 2.3 years [22].

The presence of lead time bias assumes the earlier diagnosis does not impact on the time of death. Given the LOLE measure is based on the difference between the observed time of death and the time of death for the general population, even if lead time bias is present, this difference should not be impacted by the time of diagnosis. Therefore, it is unlikely that our results can be explained by any effect of lead-time bias (Figure B.1). In order to test this hypothesis, a sensitivity analysis (using breast cancer as an example) was performed to gain an understanding of the potential impact of lead time bias on the observed results: the LOLE estimates were recalculated for female breast cancer patients by moving the date of diagnosis to five years earlier, but retaining the same death or censoring date.

All statistical analyses were performed using Stata/SE version 16 (StataCorp, TX, USA). Flexible parametric cure models were fitted using the stpm2 package [23, 24].

\section{Results}

Among the 1,865,154 cases aged 50-89, prostate cancer accounted for the largest proportion of cases $(19 \%)$ followed by colorectal cancer (16\%) and breast cancer (13\%) (Table A.1).

\subsection{Pattern for all cancers combined}

For males, females and persons, the LOLE and POLL were lower for more recently diagnosed cancers (Table 1). While the direction of this trend was consistent across age groups, the magnitude of the effect varied. For example, the POLL for persons aged 50-59 reduced from $54 \%$ in $1982-1989$ to $32 \%$ in $2010-2015$, which equates to a decrease of 5 years of LOLE. Corresponding estimates for persons aged $80-89$ were $49 \%$ and $39 \%$, a decrease of 0.1 years of LOLE.

These reductions over time were also observed for the NSW cohort stratified by spread of disease (Table 2). For the known spread of disease categories, people diagnosed with regional disease experienced the greatest absolute decrease in the POLL over the three time periods. 
Additionally, people diagnosed with more advanced disease had higher LOLE and POLL in each time period than those diagnosed with localised disease.

\subsection{Patterns for specific cancers}

Overall, the POLL generally decreased over time for all cancers except cervical cancer (Figure B.2 \& Figure B.3). Australians diagnosed with prostate, kidney, leukaemia, myeloma, or breast cancers or non-Hodgkin lymphoma (NHL) experienced the largest decrease, leading to the POLL over 34 years being more than $30 \%$ lower in absolute terms.

When considering the temporal changes by age groups (Figure $2 \&$ Figure B.4), the biggest decrease in the POLL was seen for the youngest age group (50-59 years) for most cancer types. This trend was most obvious for prostate cancer, leukaemia and myeloma.

For people diagnosed with cancers having poor prognosis, such as liver, gallbladder, and lung cancers, the largest decreases in POLL were seen for those with localised disease (Figure $3 \&$ Figure B.4). For example, the POLL for localised liver cancer reduced from $90 \%$ to $70 \%$ between 1982-1984 and 2012-2014 respectively. Corresponding estimates for distant liver cancer were $98 \%$ and $92 \%$. In contrast, for cancers with good prognosis such as breast and prostate cancers, the largest decreases over time were observed for people diagnosed with distant disease.

\subsection{Gain in life years for those diagnosed in 2014}

The 19 cancers included in this study were estimated to cause 403,094 years of LOLE for Australians diagnosed in 2014 (Figure 4). Lung, colorectal, pancreatic and female breast cancers were the four cancers with the largest LOLEs, accounting for 54\% of the total LOLE. If those patients diagnosed in 2014 had the same relative survival as those diagnosed in 1982, the estimated overall LOLE for the 2014 cohort would have been 835,682 years. That meant that 432,588 additional life years were gained by the 2014 cancer cohort due to the improvements in cancer survival over 34 years. For the overall cohort, the largest 'gains in life years' were for prostate cancer (160,478 years), followed by breast $(89,525$ years) and colorectal cancer $(46,072$ years). For individual patients, the largest gain in life years were usually seen in 50-59 age group. In this age group, the highest average gain in life years per person were observed for people diagnosed with prostate cancer (18.3 years), leukaemia (14.1 years), and NHL (13.8 years) (Table A.2).

\subsection{Sensitivity analysis}


Our sensitivity analysis for female breast cancer moving the date of diagnosis five years earlier while retaining the same death or censoring date showed that there was little quantitative impact on the LOLE (Table A.3). The overall average LOLE only changed by 0.2 years, from 3.7 to 3.5 years, with overlapping 95\% CIs. This pattern was consistent across all age-period groups.

\section{Discussion}

This study demonstrated a consistent decrease in LOLE and POLL over 34 years for 19 leading cancers in Australia. It suggests that cancer patients can, on average, anticipate a longer life expectancy than before, and this increase occurred at a greater rate than that for the general population. Due to this encouraging trend, we estimated that this cohort of Australian cancer patients will, in total, gain an extra 432,588 life years due to survival increases since 1982. To the best of our knowledge, this study is the first to quantify "gain in life years" as a measure of the population-level impact of temporal improvements in cancer survival.

The decreasing LOLE observed in this study is largely consistent with previous Australian [11] and international studies [7, 9]. However, a narrow age range for our cohort, a more contemporary time period and a wider range of cancer types compared to the previous Australian study meant that the results are not directly comparable.

While the key drivers for the decreased LOLE over time are likely to be multifactorial and the examination of these is beyond the scope of this study, some possible explanations are worth considering. The introduction of cancer screening is one potential explanation. Population-based screening programs for breast (since 1991) [25] and cervical cancer (1991) [26] have been conducted in Australia for several decades, while the continuing rollout of the bowel cancer screening program started in 2006 [27]. Although prostate specific antigen testing is not a routine screening approach for prostate cancer in Australia, ad-hoc participation by Australian men aged 50-79 years is relatively high $(21 \%-27 \%)$ [28, 29].

Cancer screening can impact on survival through several ways, including more effective treatment for early-stage cancers; lead-time bias [21]; or over-diagnosis, i.e. diagnosis of "cancers" that would never have caused harm to the patients had they remained undetected [30]. In the presence of screening it is difficult to disentangle how much of the observed improvement in standard survival measures is real and how much is due to lead-time bias [22]. The advantage of the LOLE measure is that it is more robust to lead-time bias than other 
survival measures, as illustrated by our sensitivity analyses (Table A.3). However, the LOLE estimates will still be influenced by the presence of over-diagnosis, since 'healthy' people with zero LOLE would be included in the cancer cohort, and this will reduce the combined LOLE estimates. For example, 42\% of prostate cancers diagnosed in Australia in 2012 are estimated to be due to overdiagnosis [31], most of which is attributed to the role of PSA testing introduced in the early 1990s. This is likely to explain much of the significantly reduced prostate cancer LOLE from the early 1990s.

Developments in cancer treatment over time are another potential reason for the reductions in LOLE. Examples of treatments that have resulted in survival improvements over time include the introduction of total mesorectal excision for colorectal cancer [32], increasing use of neoadjuvant and endocrine therapies for female breast cancer [33], the use of androgenablation therapy for prostate cancer [34], and the improvements in chemotherapy for blood cancers [35-37].

This study found a larger reduction in the POLL for younger age groups (aged 50-59 at diagnosis), especially for those with prostate or blood cancers. A previous Australian study also reported similar patterns [11]. It is favourable as each percentage decrease in the POLL means more life years gained for younger patients, as their population life expectancy is longer than for older patients. Possible explanations for this observed pattern may include that younger patients usually have fewer comorbidities and better renal and hepatic function to tolerate the adverse effects of treatments including chemotherapy [38].

For cancers with generally poor survival rates such as liver, gallbladder, and lung cancers, the largest reductions in the POLL were seen for those with localised disease, emphasising the importance of early diagnosis.

For those diagnosed in 2014, the LOLE caused by lung, colorectal, pancreatic and female breast cancers accounted for 54\% of the total LOLE for all cancers combined. Except for pancreatic cancer, the other three cancers are either the most preventable (lung cancer in particular [39]) or the most treatable if detected early (colorectal [40] and breast cancers [41]). Implementation of effective interventions may reduce the LOLEs for these cancers. Our study estimated that more than 430,000 life years will be gained among Australians diagnosed with cancer in 2014. Those diagnosed in their 50-59 years experienced the highest gain in life years due to the improvement of cancer-related survival since 1982. These results quantified the reduced burden of cancer in life years lost due to the improvement in relative 
survival of cancers over time. On the other hand, as cancer patients are living longer than before, there is a growing demand for best-practice survivorship care to enable cancer survivors to engage in post-treatment follow-up and self-management activities [42]. Study strengths included the use of high-quality national population-based cancer registration and mortality data. In addition, since the POLL measure has been shown to be more consistent across age at diagnosis [17], it overcomes the sensitivity of LOLE to changes in the age at diagnosis over time or between cancers. Also, we were able to report on the temporal trends in the POLL by spread of disease using NSW data.

We estimated the annual change of POLL by assuming a linear trend over time, this assumption may have oversimplified the trends in the POLL, so further extrapolations of these linear trends over time would not be recommended. In addition, while we found the effect of spread of disease on LOLE trends varied by cancer type, we cannot rule out the impact of stage migration due to improvements in diagnostic and reporting processes [43]. Finally, this study reported the temporal trend in LOLE up to 2015, with follow-up for vital status to 31 December 2015. Although this is consistent with previous studies $[9,10]$, and the life expectancy estimated using flexible parametric models with the cure assumption has been reported to be accurate with short follow-up periods [16], the estimations for those diagnosed in 2014 with follow-up of less than one year need to be interpreted cautiously.

\section{Conclusion}

The LOLE provides an additional and potentially more tangible measure of survival outcomes after a cancer diagnosis. The increased life expectancy over 34 years quantifies the impact of cancer survival improvement across the whole lifespan of a patient. The large amount of gain in life years estimated for those diagnosed more recently, although encouraging, also highlights the increasing demands on survivorship services and new challenges for the patients themselves, their care givers, communities, and health professionals. 


\section{Tables and Figures}

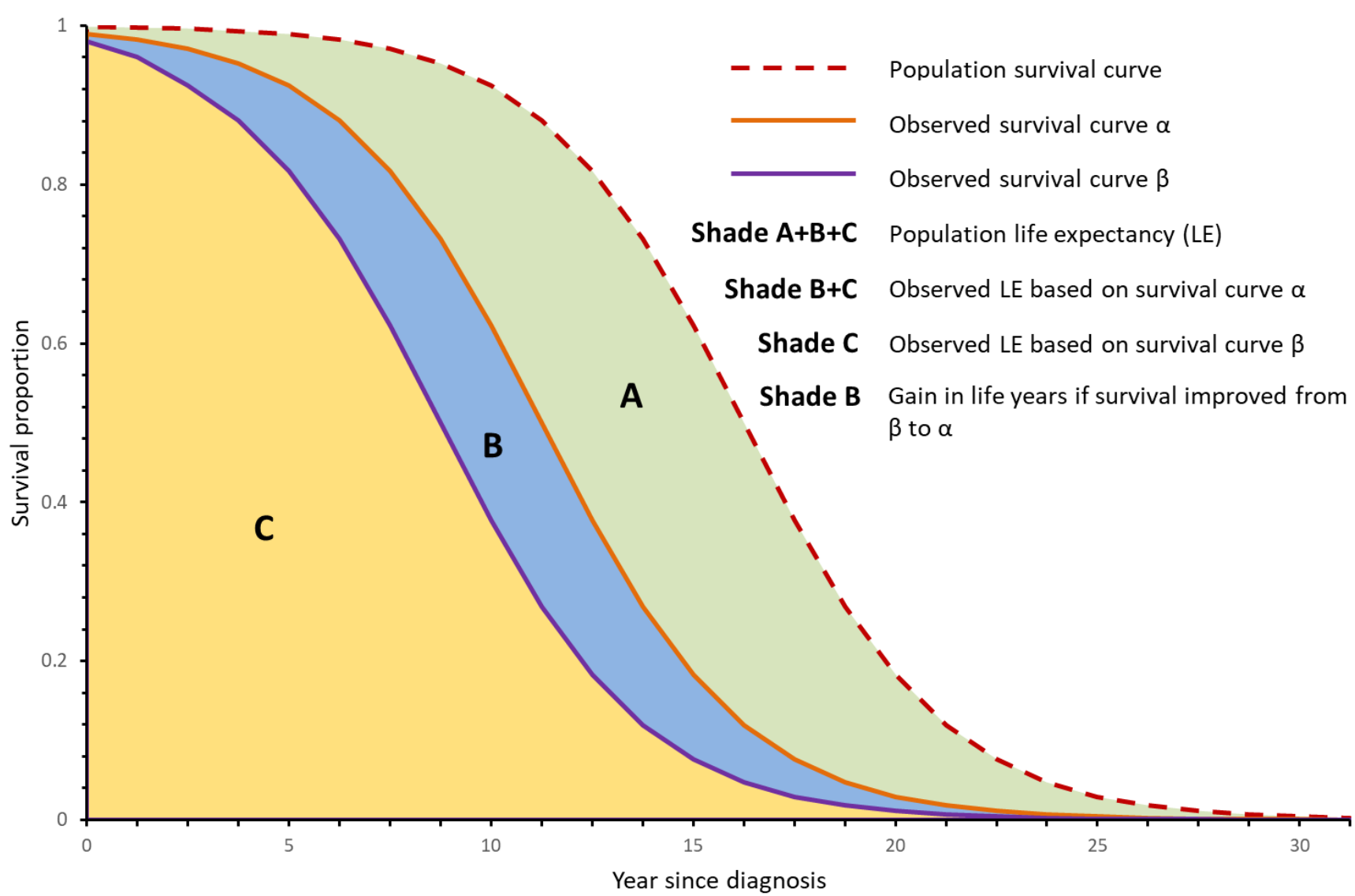

Figure 1 Example illustration of 'gain in life years' due to improvement in cancer survival.

Note: In this study for calculating gain in life years for those diagnosed in 2014, population survival curve represents the population survival in 2014; Observed survival curve $\alpha$ is based on 2014 population and relative survival estimates; Observed survival curve $\beta$ is based on 2014 population and 1982 relative survival estimates. 
Table 1 Loss of life expectancy and proportion of life lost for patients diagnosed with one of 19 cancer types combined by sex, age at diagnosis, and period of diagnosis, Australia, age at diagnosis 50-89 years, 1982-2015

\begin{tabular}{cccccccc}
\hline \multirow{2}{*}{ Sex } & Age at & \multicolumn{2}{c}{ Loss of life expectancy in years (95\% CI) } & \multicolumn{2}{c}{ Proportion of life lost in \% (95\% CI) } \\
\cline { 3 - 7 } & diagnosis & $1982-1989$ & $1990-1999$ & $2000-2015$ & $1982-1989$ & $1990-1999$ & $2000-2015$ \\
\hline \multirow{4}{*}{ Male } & $50-59$ & $14.2(13.8-14.6)$ & $11.7(11.4-11.9)$ & $9.0(8.7-9.3)$ & $58(59-56)$ & $43(44-42)$ & $32(33-31)$ \\
& $60-69$ & $8.8(8.6-9.0)$ & $7.8(7.6-7.9)$ & $6.4(6.2-6.6)$ & $54(55-53)$ & $43(44-43)$ & $33(33-32)$ \\
& $70-79$ & $4.9(4.8-5.0)$ & $4.8(4.7-4.9)$ & $4.2(4.1-4.3)$ & $52(53-51)$ & $44(45-44)$ & $35(36-34)$ \\
& $80-89$ & $2.7(2.6-2.8)$ & $2.6(2.5-2.7)$ & $2.6(2.5-2.7)$ & $51(53-49)$ & $45(46-44)$ & $39(41-38)$ \\
& $50-59$ & $14.7(14.2-15.1)$ & $12.5(12.1-12.8)$ & $9.8(9.3-10.3)$ & $49(51-48)$ & $40(41-39)$ & $31(33-30)$ \\
Female & $60-69$ & $9.9(9.7-10.1)$ & $8.8(8.6-9.0)$ & $7.3(7.0-7.6)$ & $49(50-47)$ & $41(42-40)$ & $32(34-31)$ \\
& $70-79$ & $5.8(5.7-6.0)$ & $5.5(5.4-5.6)$ & $5.1(4.8-5.3)$ & $47(49-46)$ & $42(42-41)$ & $35(37-34)$ \\
& $80-89$ & $3.1(3.0-3.2)$ & $3.0(2.9-3.1)$ & $3.0(2.8-3.1)$ & $47(49-45)$ & $42(44-41)$ & $39(41-37)$ \\
& $50-59$ & $14.3(13.9-14.7)$ & $12.0(11.7-12.3)$ & $9.3(9.0-9.7)$ & $54(55-52)$ & $42(43-41)$ & $32(33-31)$ \\
\multirow{6}{*}{ Personyyyyyy} & $60-69$ & $9.2(9.0-9.4)$ & $8.1(8.0-8.3)$ & $6.8(6.5-7.0)$ & $51(53-50)$ & $42(43-41)$ & $32(34-31)$ \\
& $70-79$ & $5.3(5.2-5.4)$ & $5.1(5.0-5.2)$ & $4.6(4.4-4.7)$ & $50(51-48)$ & $43(44-42)$ & $35(36-34)$ \\
& $80-89$ & $2.9(2.8-3.0)$ & $2.8(2.7-2.9)$ & $2.8(2.7-2.9)$ & $49(50-47)$ & $44(45-43)$ & $39(41-38)$ \\
\hline
\end{tabular}

Note: Loss of life expectancy and proportion of life lost were estimated using cancer-specific flexible parametric cure models (covariates: sex, age, calendar year of diagnosis; interaction: age and year of diagnosis). Estimates for the combined cancer types were generated by calculating a weighted average of the cancer-specific estimates, with the weights reflecting the distribution of cancer types over the whole study period for the relevant cohort. 
Table 2 Loss of life expectancy and proportion of life lost for patients diagnosed with one of 16 cancer types combined by spread of disease, age at diagnosis, and period of diagnosis, New South Wales, 1982-2014, age at diagnosis 50-89

\begin{tabular}{|c|c|c|c|c|c|c|c|}
\hline \multirow{3}{*}{ Localised } & $50-59$ & $6.4(5.9-6.9)$ & $5.3(4.9-5.6)$ & $3.9(3.6-4.2)$ & $24(23-26)$ & $19(18-20)$ & $14(12-15)$ \\
\hline & $70-79$ & $2.2(2.1-2.4)$ & $2.2(2.1-2.3)$ & $2.0(1.8-2.1)$ & $21(20-22)$ & $19(18-20)$ & $15(14-16)$ \\
\hline & $80-89$ & $1.2(1.1-1.3)$ & $1.2(1.1-1.3)$ & $1.2(1.1-1.2)$ & $20(18-22)$ & $19(17-20)$ & $16(15-18)$ \\
\hline \multirow{3}{*}{ Regional } & $50-59$ & $15.7(14.9-16.4)$ & $13.5(12.9-14.1)$ & $10.2(9.6-10.9)$ & $58(55-61)$ & $47(45-49)$ & $35(33-37)$ \\
\hline & $70-79$ & $5.7(5.5-6.0)$ & $5.7(5.5-5.9)$ & $5.0(4.8-5.3)$ & $53(51-55)$ & $47(45-49)$ & $39(37-40)$ \\
\hline & $80-89$ & $3.0(2.8-3.2)$ & $3.0(2.8-3.1)$ & $2.9(2.7-3.1)$ & $50(47-53)$ & $46(44-48)$ & $40(38-43)$ \\
\hline \multirow{4}{*}{ Distant } & $50-59$ & $24.9(24.6-25.1)$ & $25.7(25.4-26.0)$ & $23.9(23.2-24.6)$ & $94(93-95)$ & $91(90-92)$ & $82(80-85)$ \\
\hline & $60-69$ & $16.5(16.4-16.6)$ & $17.2(17.1-17.4)$ & $16.8(16.4-17.2)$ & $94(93-95)$ & $91(90-91)$ & $82(80-83)$ \\
\hline & $70-79$ & $9.6(9.6-9.7)$ & $10.6(10.5-10.7)$ & $10.8(10.7-11.0)$ & $92(91-93)$ & $90(89-91)$ & $84(83-86)$ \\
\hline & $80-89$ & $5.4(5.3-5.5)$ & $5.8(5.7-5.9)$ & $6.1(6.0-6.2)$ & $91(90-92)$ & $90(89-91)$ & $87(85-88)$ \\
\hline
\end{tabular}

Note: Loss of life expectancy and proportion of life lost were estimated using cancer-specific flexible parametric cure models (covariates: sex, age, spread of disease, calendar year of diagnosis; interaction: age and year of diagnosis). Estimates for the combined cancer types were generated by calculating a weighted average of the cancer-specific estimates, with the weights reflecting the distribution of cancer types within each spread of disease category over the whole study period for the relevant cohort. As there were no cases in certain age-year-sex-spread of disease groups for several cancers, results were averaged over the sex distribution and were not standardised by sex. 

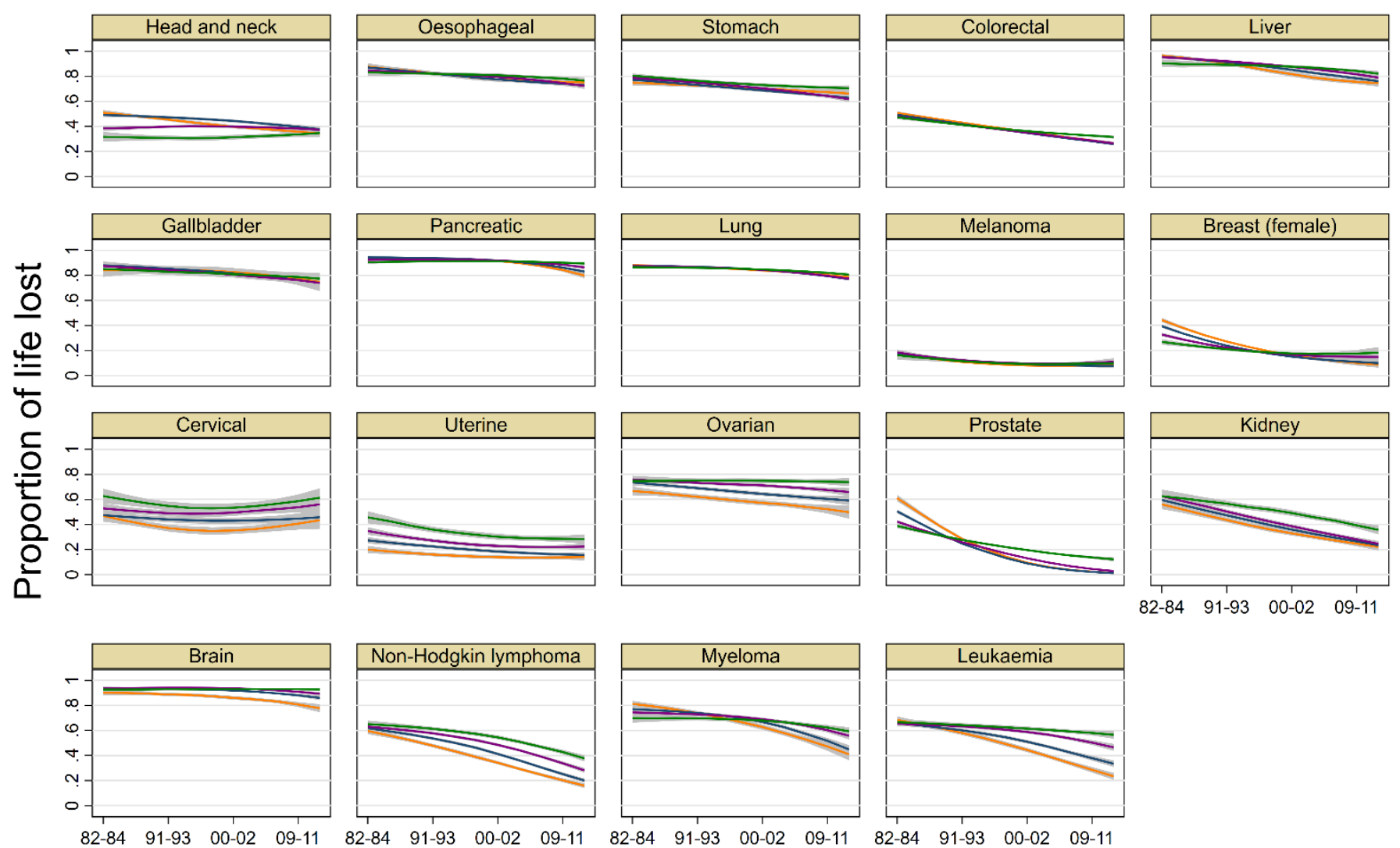

Year at diagnosis

\section{$50-59$}

60-69

$70-79$

Figure 2 Temporal trends in proportion of life lost from 1982 to 2015 by age group and cancer type in Australia, age at diagnosis $50-89$ years. Note: Proportions of life lost were combined into three-year time periods due to small numbers in each year-age group. As there were no cases in certain age-year groups for several cancers, results were averaged over the sex distribution and were not standardised by sex. 

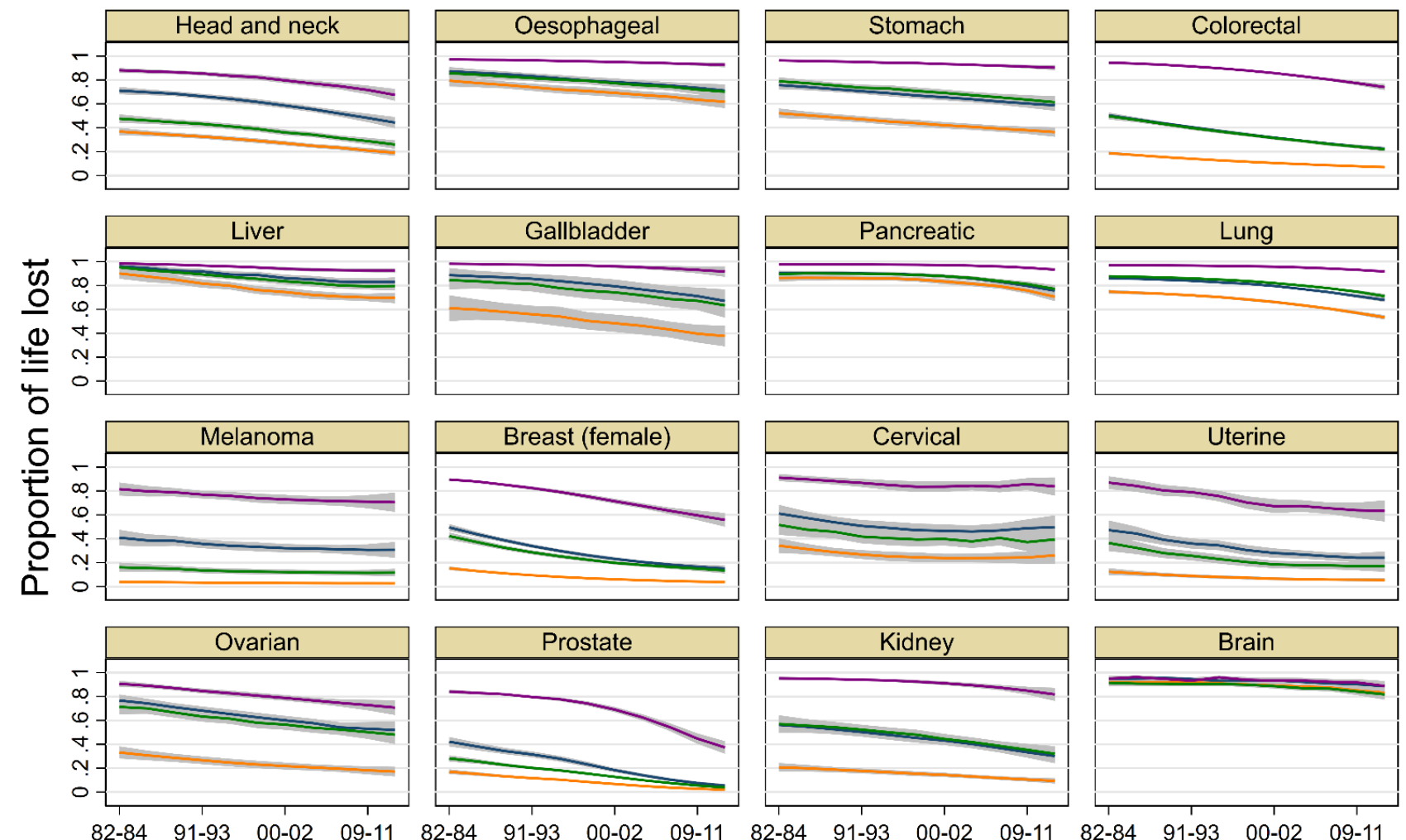

Year at diagnosis

Localised $\longrightarrow$ Regional Unknown

Figure 3 Temporal trends in proportion of life lost from 1982 to 2014 by spread of disease and cancer type in New South Wales, age at diagnosis 50-89 years.

Note: Proportions of life lost were combined into three-year time periods due to small numbers in each year-spread of disease group. Proportion of life lost was age standardised using the age distribution for the whole cohort. As there were no cases in certain age-year-sex-spread of disease groups for several cancers, results were averaged over the sex distribution and were not standardised by sex. 


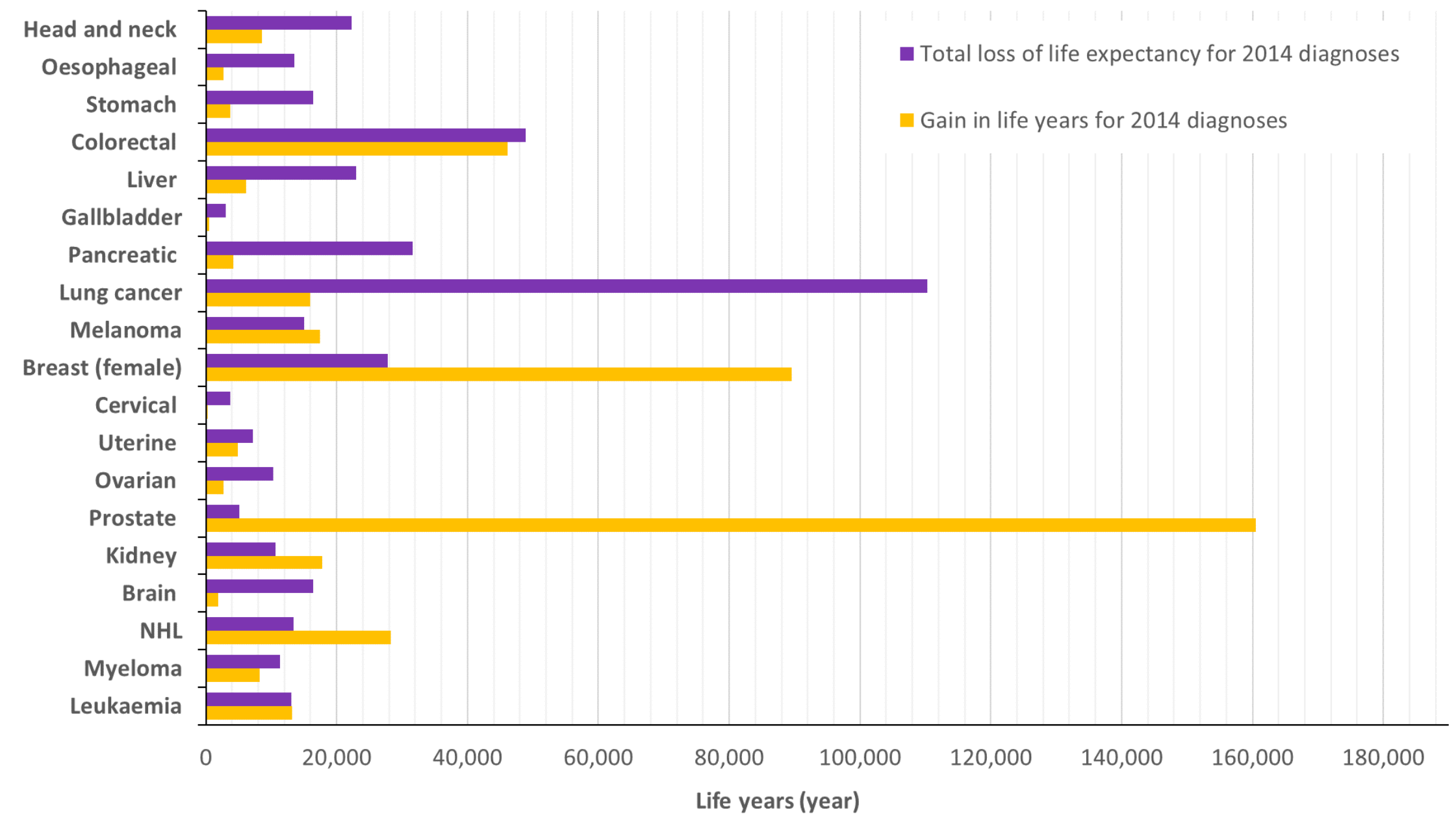

Figure 4 Total estimated loss of life expectancy and 'gain in life years' in those diagnosed in 2014, Australia, aged 50-89 years Note: 1. NHL: Non-Hodgkin lymphoma. 2. Life expectancy was estimated using flexible parametric cure models (covariates: sex, age, calendar year of diagnosis; interaction: age and year of diagnosis). 3. Total estimated loss of life expectancy for those diagnosed in 2014 is based on cancer-specific relative survival in 2014. Expected loss of life expectancy for those diagnosed in 2014 is based on 1982 relative survival. Gain in life years is the difference between the expected and estimated loss of life expectancy. 


\section{Appendix A: Supplementary tables}

Table A.1 Characteristics of study cohort, Australia, 1982-2015, age at diagnosis 50-89 years.

\begin{tabular}{lrcccr}
\hline Cancer type (ICD-10) & Cases & \% all cases & Median age (years) & \% males & \% died \\
\hline Head \& neck (C00-14, C30-32) & 83,149 & 4 & 65 & 75 & 67 \\
Oesophageal (C15) & 27,249 & 1 & 70 & 66 & 88 \\
Stomach (C16) & 50,163 & 3 & 71 & 66 & 86 \\
Colorectal (C18-20) & 303,606 & 16 & 70 & 55 & 65 \\
Liver (C22) & 21,228 & 1 & 68 & 73 & 85 \\
Gallbladder (C23) & 7,599 & 0 & 72 & 30 & 90 \\
Pancreatic (C25) & 49,877 & 3 & 71 & 52 & 93 \\
Lung (C33-34) & 226,075 & 12 & 70 & 67 & 91 \\
Melanoma (C43) & 172,693 & 9 & 66 & 60 & 41 \\
Breast (female) (C50) & 248,104 & 13 & 64 & 0 & 42 \\
Cervical (C53) & 13,048 & 1 & 64 & 0 & 70 \\
Uterine (C54-55) & 41,532 & 2 & 65 & 0 & 45 \\
Ovarian (C56) & 26,405 & 1 & 67 & 0 & 78 \\
Prostate (C61) & 362,897 & 19 & 69 & 100 & 46 \\
Kidney (C64) & 45,963 & 2 & 67 & 64 & 57 \\
Brain (C71) & 25,465 & 1 & 66 & 57 & 93 \\
NHL (C82-86) & 76,607 & 4 & 69 & 54 & 62 \\
Myeloma (C90) & 29,123 & 2 & 70 & 55 & 76 \\
Leukaemia (C91-95) & 54,371 & 3 & 70 & 59 & 72 \\
Total & $\mathbf{1 , 8 6 5 , 1 5 4}$ & $\mathbf{1 0 0}$ & $\mathbf{6 8}$ & $\mathbf{5 8}$ & 62 \\
\hline ICD: Internati
\end{tabular}

ICD: International Classification of Diseases, NHL: non-Hodgkin lymphoma. 
Table A.2 Total and average gain in life years for those diagnosed in 2014 due to the improvement of cancer-specific survival since 1982, Australia, aged 50-89 years.

\begin{tabular}{lrcccc}
\hline Cancer & Total gain in life & \multicolumn{4}{c}{ Averaged gain in life years per person (years) } \\
\cline { 3 - 6 } & years (years) & $50-59$ & $60-69$ & $70-79$ & $80-89$ \\
\hline Head \& neck & 8547.3 & 5.3 & 2.9 & 0.5 & -0.1 \\
Oesophageal & 2723.3 & 4.0 & 3.3 & 2.0 & 0.6 \\
Stomach & 3713.7 & 2.8 & 3.3 & 2.6 & 0.9 \\
Colorectal & 46071.5 & 7.8 & 5.5 & 3.2 & 1.3 \\
Liver & 6189.6 & 6.4 & 4.3 & 2.3 & 0.7 \\
Gallbladder & 536.8 & 3.0 & 3.3 & 2.0 & 0.6 \\
Pancreatic & 4196.2 & 4.4 & 2.6 & 1.0 & 0.2 \\
Lung & 15947.1 & 2.9 & 2.5 & 1.6 & 0.6 \\
Melanoma & 17416.3 & 2.9 & 2.4 & 1.4 & 0.7 \\
Breast (female) & 89524.7 & 12.3 & 7.5 & 3.2 & 0.9 \\
Cervical & 293.3 & 1.5 & 0.8 & -0.1 & 0.3 \\
Uterine & 4918.4 & 2.1 & 3.0 & 2.2 & 1.7 \\
Ovarian & 2718.6 & 5.8 & 3.8 & 1.7 & 0.1 \\
Prostate & 160478.0 & 18.3 & 11.2 & 6.0 & 2.2 \\
Kidney & 17762.1 & 10.6 & 8.3 & 5.7 & 2.2 \\
Brain & 1850.4 & 3.9 & 1.7 & 0.7 & 0.0 \\
NHL & 28244.0 & 13.8 & 9.6 & 5.3 & 2.3 \\
Myeloma & 8243.9 & 12.5 & 7.6 & 3.0 & 0.9 \\
Leukaemia & 13213.2 & 14.1 & 7.4 & 3.0 & 0.9 \\
\hline
\end{tabular}

NHL: non-Hodgkin lymphoma. 
Table A.3 Estimated loss of life expectancy and predicted loss of life expectancy if patients diagnosed 5 years earlier for breast cancer, Australia, 1987-2015, aged 50-89

\begin{tabular}{ccccccc}
\hline \multirow{2}{*}{$\begin{array}{c}\text { Age at } \\
\text { diagnosis }\end{array}$} & \multicolumn{5}{c}{ Loss of life expectancy in years (95\% CI) } \\
\cline { 2 - 7 } & Estimated & $\begin{array}{c}\text { Diagnosed 5 } \\
\text { years earlier* }\end{array}$ & Estimated & $\begin{array}{c}\text { Diagnosed 5 } \\
\text { years earlier }\end{array}$ & Estimated & $\begin{array}{c}\text { Diagnosed 5 } \\
\text { years earlier }\end{array}$ \\
\hline $50-59$ & $8.6(8.3-9.0)$ & $8.5(8.1-8.8)$ & $5.1(4.8-5.5)$ & $5.1(4.6-5.5)$ & $3.4(2.4-4.4)$ & $3.5(2.0-5.0)$ \\
$60-69$ & $5.3(5.1-5.5)$ & $5.0(4.8-5.2)$ & $3.4(3.2-3.6)$ & $3.3(3.0-3.5)$ & $3.0(2.1-4.0)$ & $3.0(1.7-4.4)$ \\
$70-79$ & $2.9(2.8-3.0)$ & $2.5(2.4-2.6)$ & $2.3(2.2-2.5)$ & $2.0(1.8-2.1)$ & $2.2(1.7-2.8)$ & $1.6(1.2-2.1)$ \\
$80-89$ & $1.5(1.4-1.6)$ & $1.0(0.9-1.0)$ & $1.3(1.2-1.4)$ & $0.9(0.8-0.9)$ & $1.5(1.2-1.8)$ & $1.0(0.7-1.3)$ \\
\hline
\end{tabular}

*Predicted loss of life expectancy if patients diagnosed 5 years earlier was estimated by moving each patient's date of diagnosis and age of diagnosis five years forward (e.g. a woman diagnosed at 50 years in 1987 was set as diagnosed at 45 years in 1982 but the death/censoring date remained unchanged). The 1982-1986 cohort was excluded from this analysis since the population mortality data were only available for the period 1982-2015. 


\section{Appendix B: Supplementary figures}

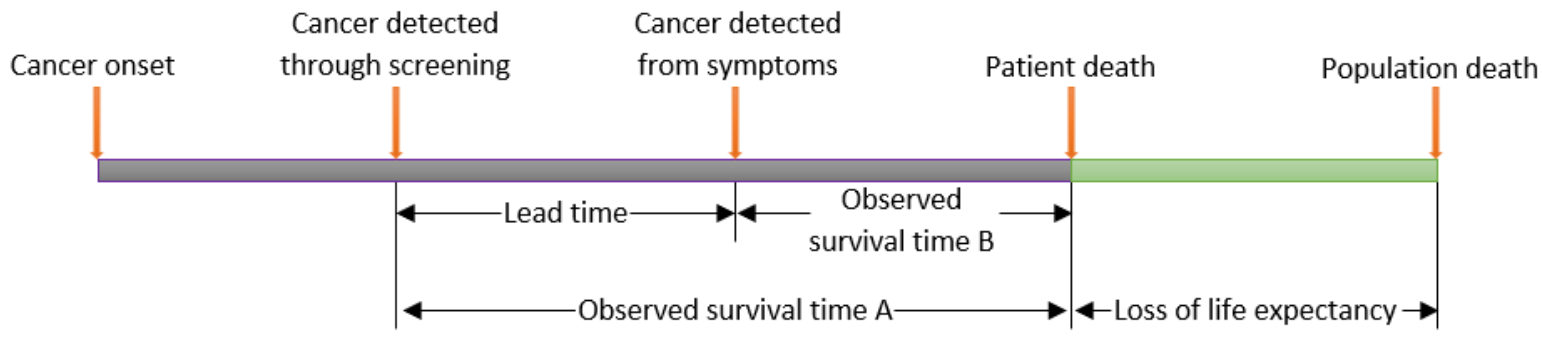

Figure B.1 An illustration of the influence of lead-time bias on loss of life expectancy.

Note: The loss of life expectancy will not be influenced by lead-time bias, as the difference between patient death and population death does not change with the time point of cancer detection, if the early detection through screening did not change the progression of the disease. 

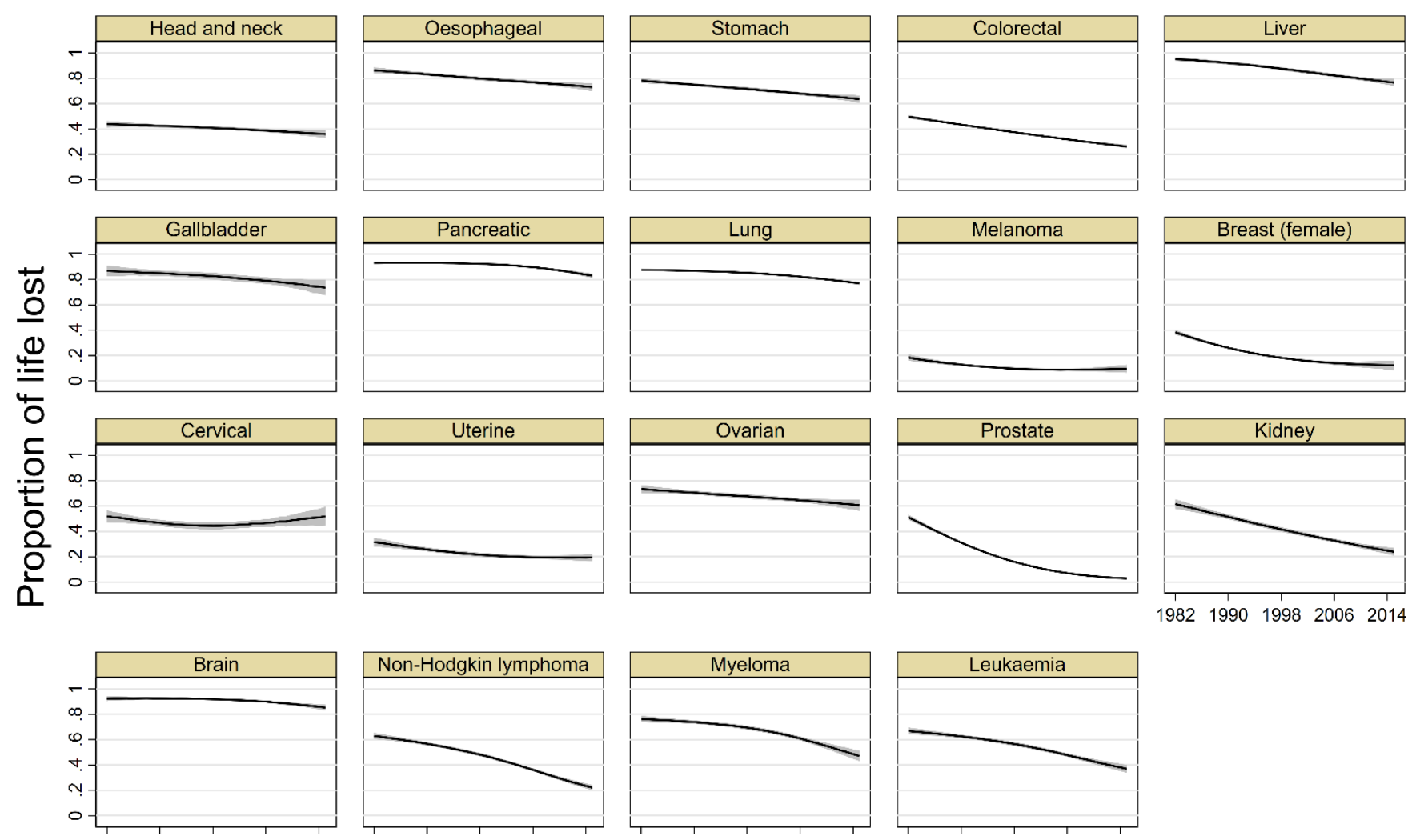

19821990199820062014

19821990199820062014198219901998200620141982199019982006201419821990199820062014

\section{Year at diagnosis}

\section{$95 \% \mathrm{Cl} \longrightarrow$ Proportion of life lost}

Figure B. 2 Temporal trends in proportion of life lost from 1982 to 2015 by cancer type in Australia, age at diagnosis 50-89 years.

Note: Proportion of life lost was calculated as the patients' loss of life expectancy divided by population life expectancy; Loss of life expectancy and population life expectancy were estimated using flexible parametric cure models (covariates: sex, age, calendar year of diagnosis; interaction: age and year of diagnosis). Proportion of life lost was age standardised using the age distribution for the whole cohort. As there were no cases in certain age-year-sex groups for several cancers, results were averaged over the sex distribution and were not standardised by sex. 


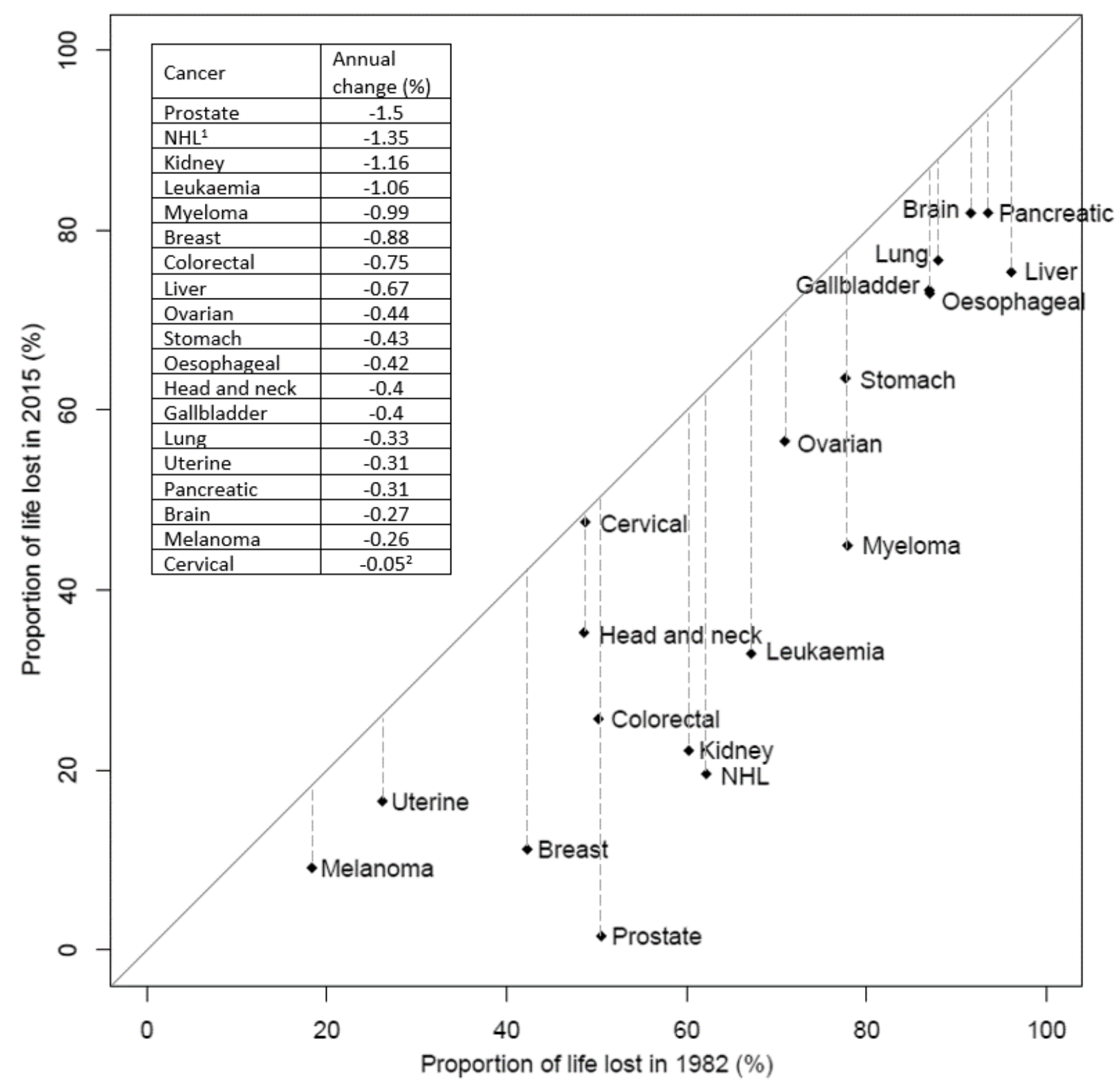

Figure B.3 Proportion of life lost in 1982 and 2015 and the average annual change (\%) in proportion of life lost.

Note: 1.NHL: non-Hodgkin lymphoma; 2. Annual changes in proportion of life lost were assessed and estimated using linear regression models (dependent variable is the 1982-2015 year-specific proportion of life lost; independent variable is year of diagnosis). All the p-values were $<0.001$ except cervical cancer $(p=0.293)$; Proportion of life lost was calculated as the patient loss of life expectancy divided by the population life expectancy; Loss of life expectancy and population life expectancy were estimated using flexible parametric cure models (covariates: sex, age, calendar year of diagnosis; interaction: age and year of diagnosis). 3. Solid line represents $x=y$. For cancers below the solid line, plotted estimates indicate a smaller proportion of life lost in 2015 than in 1982; 4. Dashed lines represent the difference in the proportion of life lost between 1982 and 2015. 


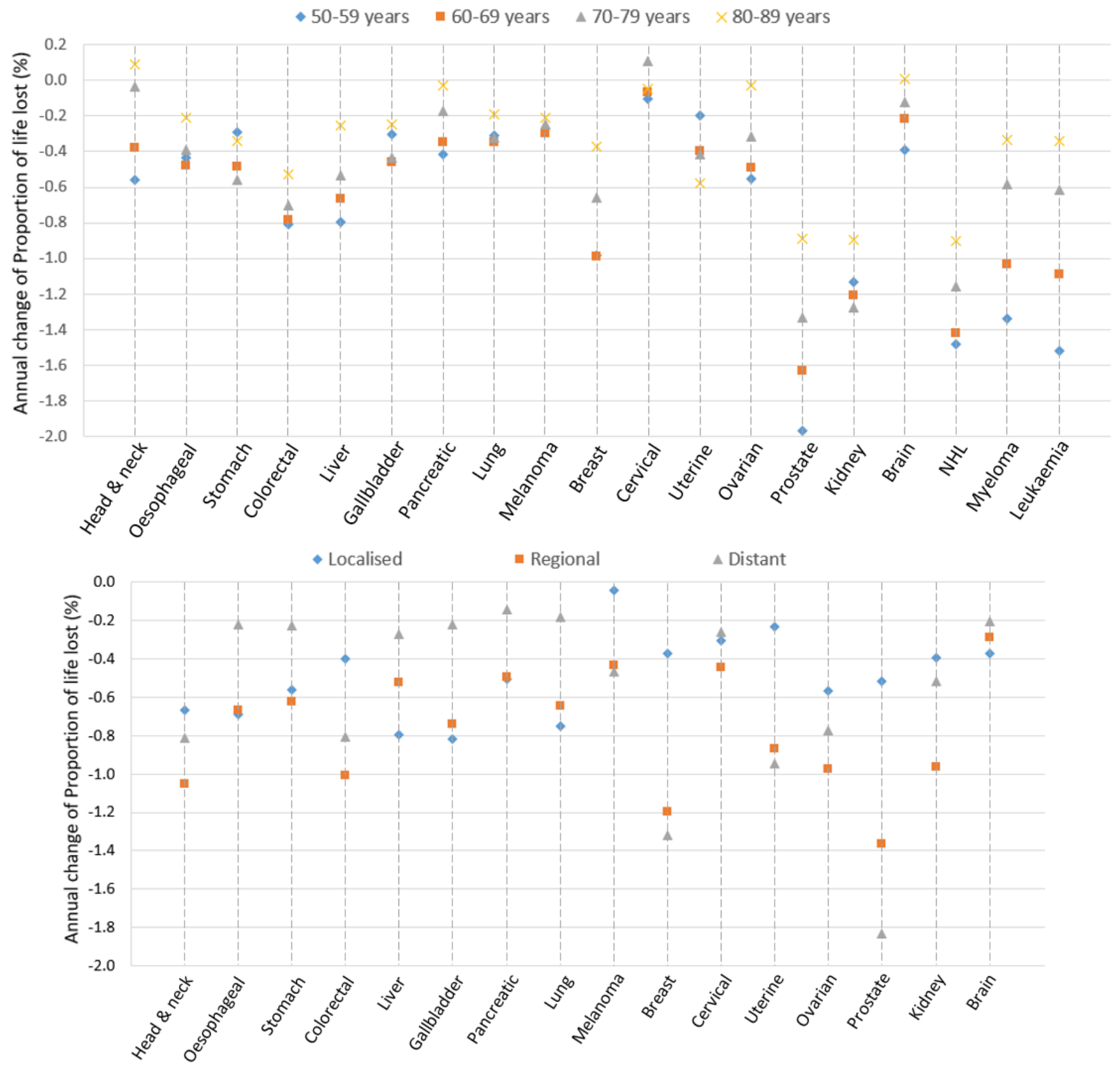

Figure B.4 Annual change in proportion of life lost by age groups, Australia, 1982-2015 (upper), and by spread of disease, New South Wales, 1982-2014 (lower), age at diagnosis 5089 years.

Note: 1. Proportion of life lost was calculated as the patients' loss of life expectancy divided by population life expectancy; Loss of life expectancy and population life expectancy were estimated using flexible parametric cure models (covariates: sex, age, calendar year of diagnosis, and for New South Wales data only, spread of disease; interaction: age and year of diagnosis). 2. Annual changes were estimated using linear regression models (dependent variable is the year-specific proportion of life lost; independent variable is year of diagnosis). 


\section{Appendix C: Code for 'gain in life years' estimation}

* Melanoma example

* Estimate gain in life years in 1994 cohort, due to the improvement of relative survival from 1975 to 1994

$* * * * * * * * * * * * * * * * * * * * * * * * * * * * * * * * *$

/* Step 1: population life expectancy */

/* Equals to shaded area $\mathrm{C}$ in Figure $1 * /$

$* * * * * * * * * * * * * * * * * * * * * * * * * * * * * * * * *$

do http://www.pauldickman.com/survival/install_packages.do /*skip if you already have this installed*/

cd "C:Isurvival"

use melanoma, clear

stset surv_mm, failure(status=1 2) scale(12) exit(time 120.5) id(id)

keep if inrange(age, 50, 89)

/* match with population mortality rate */

qui gen_age $=\min \left(\operatorname{int}\left(\right.\right.$ age $+_{-}$t $\left.), 99\right)$

qui gen_year $=\operatorname{int}\left(\mathrm{yydx}+\_\mathrm{t}\right)$

qui sort_year sex_age

qui merge m:1_year sex_age using popmort, keep(match master) keepusing(rate)

qui drop_age _year_merge

/* Use splines for age, $\mathrm{df}=4 *$ /

qui rcsgen age, gen(rcsage) df(4) orthog

/*Use splines for year, $\mathrm{df}=2 * /$

qui rcsgen yydx, gen(rcsyear) df(2) orthog

$/ *$ generate interactions between age and year*/

forvalues $\mathrm{j}=1 / 4$ \{

forvalues $i=1 / 2\{$

gen yearage_' $i{ }^{\prime}{ }^{\prime} j$ '=rcsage ${ }^{\prime}{ }^{\prime}$ 'rcsyear ${ }^{\prime}{ }^{\prime}$

\}

$/ *$ model using $\mathrm{df}=8, \mathrm{dftvc}=3 * /$

stpm 2 rcsage* rcsyear* yearage* i.sex, scale(hazard) df(8) bhazard(rate) tvc(rcsage* rcsyear* yearage*) dftvc(3)

* use 1975 relative survival and 1994 population survival to estimate the life expectancy in 1994 if the relative survival of melanoma did not improve since 1975.

* Since the model uses cubic splines of yydx to estimate relative survival, need to get the values of cubic spline for $\mathrm{yydx}=1975$

summ rcsyear 1 if $y y d x==1975$

scalar mean1975_1='r(mean)'

summ rcsyear 2 if $y y d x==1975$

scalar mean1975_2='r(mean)'

/* keep 1994 diagnoses, change their rcsyear* to 1975 rcsyear */

keep if $y y d x==1994$

*change cubic spline of yeardx to 1975 value 
replace rcsyear $1=$ mean 1975

replace rcsyear $2=$ mean 19752

*change interactions between age and yydx(1994) to age*yydx(1975)

forvalues $\mathrm{j}=1 / 4$ \{

replace yearage_1_ ${ }^{\prime}{ }^{\prime}=$ rcsage ${ }^{\prime} \mathrm{j}^{\prime *}$ mean $1975 \_1$

replace yearage_2_'j'=rcsage ' $\mathrm{j}^{\prime *}$ mean1975_2

\}

* In this prediction of life expectancy, the relative survival predicted by the flexible parametric model will be the 1975 relative survival.

* Since the population mortality is still merged by yydx, which is still 1994, the observed survival curve will be: observed survival = relative survival (1975) x population survival (1994)

predict exp 11, lifelost mergeby( year sex age) diagage(age) ///

diagyear(yydx) nodes(40) tinf(80) using(popmort) ///

stub(exp_surv) maxyear(2000) tcond $(0) / *{ }^{*} i^{*} /$

save "predict1994_1975RS", replace

/* Total population life expectancy in $1994 *$ /

collapse (sum) exp_survobs exp_survexp exp_1l

gen flag $=1$

save LE1994_1975RS, replace

$* * * * * * * * * * * * * * * * * * * * * * * * * * * * * * * *$

/* Step 2: estimated life expectancy */

/* Equals to shaded $\mathrm{B}+\mathrm{C}$ in Figure 1 */

$* * * * * * * * * * * * * * * * * * * * * * * * * * * * * * * *$

use melanoma, clear

stset surv_mm, failure(status=1 2) scale(12) exit(time 120.5) id(id)

keep if inrange(age, 50, 89)

/* match with populaiton mortality rate */

qui gen_age $=\min ($ int $($ age + t $), 99)$

qui gen_year $=\operatorname{int}\left(\mathrm{yydx}+\_t\right)$

qui sort_year sex_age

qui merge m:1_year sex _age using popmort, keep(match master) keepusing(rate)

qui drop_age_year_merge

/* Use splines for age, $\mathrm{df}=4$ */

qui rcsgen age, gen(rcsage) df(4) orthog

/* Use splines for year, $\mathrm{df}=2 * /$

qui rcsgen yydx, gen(rcsyear) df(2) orthog

/* generate interactions between age and year*/

forvalues $j=1 / 4$ \{

forvalues $i=1 / 2\{$

gen yearage_ ${ }^{\prime}{ }^{\prime}{ }^{\prime} j$ '=rcsage ${ }^{\prime}{ }^{\prime} *$ rcsyear ${ }^{\prime} i '$

\}

\}

$/ *$ model using $\mathrm{df}=8, \mathrm{dftvc}=3 * /$ 
stpm2 rcsage* rcsyear* yearage* i.sex, scale(hazard) df(8) bhazard(rate) tvc(rcsage* rcsyear* yearage*) dftvc(3)

\section{/* keep 1994 diagnoses */}

keep if $\mathrm{yydx}==1994$

predict est_1l, lifelost mergeby(_year sex_age) diagage(age) ///

diagyear(yydx) nodes(40) tinf(80) using(popmort) ///

stub(est_surv) maxyear(2000) tcond $(0) / * c^{*}{ }^{*} /$

save "predict1994_1994RS", replace

/* Total estimated life expectancy in $1994 * /$

collapse (sum) est_survobs est_survexp est_11

gen flag $=1$

save LE1994_1994RS, replace

\section{$* * * * * * * * * * * * * * * * * * * * * * * * * * * * * * * * *$}

/* Step 3: Gain in life years $\quad * /$

/* Equals to shaded area B in Figure $1 * /$

$* * * * * * * * * * * * * * * * * * * * * * * * * * * * * * * * *$

merge 1:1 flag using LE1994_1975RS

drop_merge flag

*gain in life years $($ shade $B)=$ est_survobs $($ shade $B+C)$ - exp_survobs (shade C)

gen gain = est_survobs-exp_survobs /* in total of 812 life years will be saved for those diagnosed in 1994, thanks to the improvement of relative survival from 1975 to 1994 . */

label variable est_survexp "Total population life expectancy in 1994 diagnoses, if they were not diagnosed with cancer"

label variable exp_survexp "Total population life expectancy in 1994 diagnoses, if they were not diagnosed with cancer"

label variable est_survobs "Total observed life expectancy in 1994 diagnosis"

label variable exp_survobs "Total observed life expectancy in 1994 diagnosis, if they had the same relative survival with those diagnosed in 1975"

label variable est_ll "Total loss of life expectancy in 1994 diagnosis"

label variable exp_1l "Total loss of life expectancy in 1994 diagnosis, if they had the same relative survival with those diagnosed in 1975"

label variable gain "Total gain in life years due to the improvement of relative survival from 1975 to 1994"

format \%9.0f est_survobs est_survexp est_ll exp_survobs exp_survexp exp_11 gain list in 1 


\section{References}

[1] J. Esteve, E. Benhamou, M. Croasdale, L. Raymond, Relative survival and the estimation of net survival: elements for further discussion, Stat. Med. 9(5) (1990) 529-538.

[2] Australian Institute of Health and Welfare 2019. Cancer in Australia 2019. Cancer series no.119. Cat. no. CAN 123. Canberra: AIHW.

[3] S. Viscomi, G. Pastore, E. Dama, L. Zuccolo, N. Pearce, F. Merletti, C. Magnani, Life expectancy as an indicator of outcome in follow-up of population-based cancer registries: the example of childhood leukemia, Ann. Oncol. 17(1) (2005) 167-171.

[4] P.D. Baade, D.R. Youlden, T.M. Andersson, P.H. Youl, M.G. Kimlin, J.F. Aitken, R.J. Biggar, Estimating the change in life expectancy after a diagnosis of cancer among the Australian population, BMJ open 5(4) (2015) e006740.

[5] P.-H. Liu, J.-D. Wang, N.L. Keating, Expected years of life lost for six potentially preventable cancers in the United States, Prev. Med. 56(5) (2013) 309-313.

[6] T.M.-L. Andersson, P.W. Dickman, S. Eloranta, A. Sjövall, M. Lambe, P.C. Lambert, The loss in expectation of life after colon cancer: a population-based study, BMC Cancer 15(1) (2015) 412.

[7] M. Bjorkholm, H. Bower, P.W. Dickman, P.C. Lambert, M. Höglund, T.M.-L. Andersson, Temporal Trends in Chronic Myeloid Leukemia Outcome Using the Loss in Expectation of Life: A Swedish Population-Based Study, Blood 126(23) (2015) 2779-2779.

[8] C.C. Huang, C.Y. Ou, W.T. Lee, J.R. Hsiao, S.T. Tsai, J.D. Wang, Life expectancy and expected years of life lost to oral cancer in Taiwan: a nation-wide analysis of 22,024 cases followed for 10 years, Oral Oncol. 51(4) (2015) 349-354.

[9] H. Bower, M. Björkholm, P.W. Dickman, M. Höglund, P.C. Lambert, T.M.-L. Andersson, Life expectancy of patients with chronic myeloid leukemia approaches the life expectancy of the general population, J. Clin. Oncol. 34(24) (2016) 2851-2857.

[10] E. Syriopoulou, H. Bower, T.M. Andersson, P.C. Lambert, M.J. Rutherford, Estimating the impact of a cancer diagnosis on life expectancy by socio-economic group for a range of cancer types in England, Br. J. Cancer 117(9) (2017) 1419-1426.

[11] P.D. Baade, D.R. Youlden, T.M. Andersson, P.H. Youl, E.T. Walpole, M.G. Kimlin, J.F. Aitken, R.J. Biggar, Temporal changes in loss of life expectancy due to cancer in Australia: a flexible parametric approach, Cancer Causes Control 27(8) (2016) 955-964.

[12] AIHW., Cancer in Australia: Actual incidence data from 1982 to 2013 and mortality data from 1982 to 2014 with projections to 2017, Asia. Pac. J. Clin. Oncol. 14(1) (2018) 5-15.

[13] T.M.-L. Andersson, M.J. Rutherford, P.C. Lambert, Illustration of different modelling assumptions for estimation of loss in expectation of life due to cancer, BMC Med. Res. Methodol. 19(1) (2019) 145.

[14] H. Barraclough, S. Morrell, M. Arcorace, H.J. McElroy, D.F. Baker, Degree-of-spread artefact in the New South Wales Central Cancer Registry, Aust. N. Z. J. Public Health 32(5) (2008) 414-416.

[15] D.E. Henson, L.A. Ries, The relative survival rate, Cancer 76(10) (1995) 1687-1688.

[16] T.M. Andersson, P.W. Dickman, S. Eloranta, M. Lambe, P.C. Lambert, Estimating the loss in expectation of life due to cancer using flexible parametric survival models, Stat. Med. 32(30) (2013) 5286-5300. 
[17] M.J. Rutherford, T.M. Andersson, M. Björkholm, P.C. Lambert, Loss in life expectancy and gain in life years as measures of cancer impact, Cancer Epidemiol. 60 (2019) 168-173.

[18] E. Syriopoulou, S.I. Mozumder, M.J. Rutherford, P.C. Lambert, Robustness of individual and marginal model-based estimates: A sensitivity analysis of flexible parametric models, Cancer epidemiol. 58 (2019) 17-24.

[19] K. Kou, P. Dasgupta, S.M. Cramb, X.Q. Yu, P.D. Baade, Temporal trends in populationlevel cure of cancer: the Australian context, Cancer Epidemiol. Biomarkers Prev. (2020).

[20] X. Yu, R. De Angelis, T. Andersson, P. Lambert, D. O’Connell, P. Dickman, Estimating the proportion cured of cancer: some practical advice for users, Cancer epidemiol. 37(6) (2013) 836-842.

[21] R.J. Jansen, B.H. Alexander, K.E. Anderson, T.R. Church, Quantifying lead-time bias in risk factor studies of cancer through simulation, Ann. Epidemiol. 23(11) (2013) 735-41.

[22] T. Andersson, M.J. Rutherford, K. Humphreys, Assessment of lead-time bias in estimates of relative survival for breast cancer, Cancer Epidemiol. 46 (2017) 50-56.

[23] P. Royston, P.C. Lambert, Flexible parametric survival analysis using Stata: beyond the Cox model, Stata College Station, Texas 2011.

[24] M. Schemper, T.L. Smith, A note on quantifying follow-up in studies of failure time, Control Clin. Trials. 17(4) (1996) 343-346.

[25] Australian Institute of Health and Welfare, BreastScreen Australia monitoring report 2012-2013, 2015.

[26] Australian Institute of Health and Welfare, Cervical screening in Australia 2014-2015, 2017.

[27] Australian Institute of Health and Welfare. Analysis of bowel cancer outcomes for the National Bowel Cancer Screening Program: 2018. Cat. no. CAN 113. Canberra: AIHW.

[28] P.D. Baade, D.R. Youlden, M.D. Coory, R.A. Gardiner, S.K. Chambers, Urban-rural differences in prostate cancer outcomes in Australia: what has changed?, Med. J. Aust. 194(6) (2011) 293-296.

[29] D.P. Smith, B.K. Armstrong, Prostate-specific antigen testing in Australia and association with prostate cancer incidence in New South Wales, Med. J. Aust. 169(1) (1998) 17-20.

[30] H.G. Welch, W.C. Black, Overdiagnosis in cancer, J. Natl. Cancer Inst. 102(9) (2010) 605-613.

[31] P.P. Glasziou, M.A. Jones, T. Pathirana, A.L. Barratt, K.J. Bell, Estimating the magnitude of cancer overdiagnosis in Australia, Med. J. Aust. (2019).

[32] S. Delibegovic, Introduction to Total Mesorectal Excision, Med. Arch. 71(6) (2017) 434.

[33] R. Van Ewijk, L. Schwentner, A. Wöckel, J. König, R. Kreienberg, M. Blettner, B.s. group, Trends in patient characteristics, treatment and survival in breast cancer in a nonselected retrospective clinical cohort study of 2,600 patients, Arch. Gynecol. Obstet. 287(1) (2013) 103-110.

[34] S.R. Denmeade, J.T. Isaacs, Development of prostate cancer treatment: the good news, Prostate 58(3) (2004) 211-224. 
[35] H. Kantarjian, S. O'brien, J. Cortes, W. Wierda, S. Faderl, G. Garcia-Manero, J.P. Issa, E. Estey, M. Keating, E.J. Freireich, Therapeutic advances in leukemia and myelodysplastic syndrome over the past 40 years, Cancer 113(S7) (2008) 1933-1952.

[36] X.Q. Yu, W.H. Chen, D.L. O'Connell, Improved survival for non-Hodgkin lymphoma patients in New South Wales, Australia, BMC cancer 10(1) (2010) 231.

[37] S.K. Kumar, S.V. Rajkumar, A. Dispenzieri, M.Q. Lacy, S.R. Hayman, F.K. Buadi, S.R. Zeldenrust, D. Dingli, S.J. Russell, J.A. Lust, Improved survival in multiple myeloma and the impact of novel therapies, Blood 111(5) (2008) 2516-2520.

[38] I. Olver, Chemotherapy for elderly patients with advanced cancer: is it worth it?, Aust. Prescr. 23(4) (2000) 80-81.

[39] M.J. Kelley, D.C. McCrory, Prevention of lung cancer: summary of published evidence, Chest. 123(1) (2003) 50S-59S.

[40] A. Costas-Chavarri, S. Temin, M.A. Shah, Treatment of Patients With Early-Stage Colorectal Cancer: ASCO Resource-Stratified Guideline Summary, J. Oncol. Pract. 15(5) (1999) 290-292.

[41] M. Cianfrocca, L.J. Goldstein, Prognostic and predictive factors in early-stage breast cancer, Oncologist. 9(6) (2004) 606-616.

[42] J.L.Vardy, R.J. Chan, B. Koczwara, K. Lisy, R.J. Cohn, D. Joske, H.M. Dhillon, M. Jefford, Clinical Oncology Society of Australia position statement on cancer survivorship care, Aust. J. Gen. Pract. 48(12) (2019) 833-836.

[43] M.P. Sormani, The Will Rogers phenomenon: the effect of different diagnostic criteria, J. Neurol. Sci. 287 (2009) S46-S49. 